Nicole Boireau, Jean-Frédéric Chevalier, Pierre Degott, éds, Réécriture des sources biographiques et construction du personnage de théâtre

Metz, Éd. Centre Écritures, coll. Recherches en littérature, 2010

\title{
Laurence Beck-Chauvard
}

\section{(2) OpenEdition \\ Journals}

\section{Édition électronique}

URL : http://journals.openedition.org/questionsdecommunication/333

DOI : 10.4000/questionsdecommunication.333

ISSN : 2259-8901

\section{Éditeur}

Presses universitaires de Lorraine

Édition imprimée

Date de publication : 30 juin 2011

Pagination : 295-296

ISBN : 978-2-8143-0084-2

ISSN : 1633-5961

\section{Référence électronique}

Laurence Beck-Chauvard, « Nicole Boireau, Jean-Frédéric Chevalier, Pierre Degott, éds, Réécriture des sources biographiques et construction du personnage de théâtre », Questions de communication [En ligne], 19 | 2011, mis en ligne le 19 avril 2012, consulté le 21 septembre 2020. URL : http://

journals.openedition.org/questionsdecommunication/333; DOI : https://doi.org/10.4000/ questionsdecommunication.333

Ce document a été généré automatiquement le 21 septembre 2020.

Tous droits réservés 


\title{
Nicole Boireau, Jean-Frédéric Chevalier, Pierre Degott, éds, Réécriture des sources biographiques et construction du personnage de théâtre
}

\author{
Metz, Éd. Centre Écritures, coll. Recherches en littérature, 2010 \\ Laurence Beck-Chauvard
}

\section{RÉFÉRENCE}

Nicole Boireau, Jean-Frédéric Chevalier, Pierre Degott, éds, Réécriture des sources biographiques et construction du personnage de théâtre, Metz, Éd. Centre Écritures, coll. Recherches en littérature, 2010, 322 p.

Les 16 textes réunis par Nicole Boireau, Jean-Frédéric Chevalier et Pierre Degott révèlent la très grande richesse d'un domaine dramatique trop rapidement jugé réducteur ou restrictif, le théâtre biographique. La problématique retenue, le traitement de la source biographique et son rapport à la construction du personnage sont explorés à partir d'un corpus d'œuvres contemporaines des $\mathrm{XX}^{\mathrm{e}}$ et du XXI ${ }^{\mathrm{e}}$ siècles, auxquelles s'ajoutent quelques pièces du XIX siècle. Majoritairement européennes et américaines - un seul article de Laurence Denooz (p. 9-31) est consacré à trois pièces égyptiennes de Tawfiq al-Hakim -, elles présentent des formes dramatiques diverses théâtre, opéra, docudrame, émission radiophonique - et mettent en scène trois types de personnages: historiques au sens plein, ceux de nos livres d'histoire: Cléopâtre, Julien l'Apostat, Macbeth, Mary Stuart et Elizabeth I⿳亠丷厂re, Luther, Napoléon, Lincoln ; contemporains : Albert Speer, l'architecte de Hitler, Harvey Milk, le héros de la cause gay, Roberto Succo, le serial killer; et des artistes: le compositeur tudor Taverner, Adrienne Lecouvreur, Giuseppe Verdi, Arthur Rimbaud. En fin de volume, un résumé en français et en anglais de chacun des articles permet de se situer aisément dans cette 
multitude de noms et d'œuvres. Aucun sentiment d'éparpillement n'entache d'ailleurs le recueil, tant la matière biographique informe le travail de création comme son analyse structure les critiques. Les sources - premières ou secondaires - et leur traitement confrontent l'auteur aux questions de l'illusion et de la vérité, aux notions de mimesis et de réécriture. Des choix s'imposent, que ce soit celui du strict respect de la masse documentaire, comme le fit David Edgar dans Albert Speer en 2000, au nom de la quête de la vérité, mais au détriment, parfois, de la clarté, ou celui de l'élagage, voire de la déformation, comme dans l'opéra engagé Harvey Milk. Anne-Sylvie Barthel-Calvet (pp. 259-274) montre comment Stewart Wallace et Michael Korie prirent le risque de la simplification pour provoquer l'adhésion du public à leur double dénonciation de l'homophobie et de l'antisémitisme et ériger Milk en emblème. L'analyse du traitement des sources constitue donc une voie d'accès privilégiée à la volonté de l'auteur, à son projet, à l'image qu'il veut donner de son protagoniste, homme réel devenu être de fiction, aux idées que met en scène l'œuvre. Ces projets s'organisent autour de trois grands axes non exclusifs. Le premier met en scène le passé pour parler du présent: Régis Debray dans sa pièce Julien le Fidèle ou Le banquet des démons publiée en 2005, invite, comme le montre Alain Cullière (pp.33-68), à une méditation sur notre temps fragilisé et dérouté. Les trois pièces de Tawfiq al-Hakim consacrées à Cléopâtre (La Nuit de noces, 1949 ; La Canne d'al-Hakim, 1955 ; Le Jeu de la mort, 1957) s'arrachent aux sources romano-centrées pour faire de la reine le symbole de l'identité nationale égyptienne. De même, dans l'opéra Hary Janos de Kodàly (1926), présenté par Thierry Santurenne (pp.159-175), Napoléon est le repoussoir symbolique contre lequel se construit le sentiment national hongrois. Quant au Gloriana de Benjamin Britten, commandé pour le couronnement d'Elizabeth II, il tend, nous rappelle Gilles Couderc (pp. 113-128), à exalter la fierté nationale et à faire renaître l'opéra anglais. Entièrement construite autour de la question «Speer connaissait-il la vérité sur la Solution finale? ", la pièce de David Edgar oblige à un travail de mémoire. Bien plus, Marianne Drugeon (pp. 237-258) souligne comment l'auteur convoque le terme de catharsis pour donner sens à sa représentation du Mal.

2 D'autres œuvres sont principalement concernées par un questionnement psychologique, celui de l'identité du protagoniste comme celui de l'auteur. Ainsi les six opéras sur Rimbaud composés de 1978 à 2007 et étudiés par Doriane Bier (pp. 219-235) se concentrent-ils davantage sur la fragmentation du Moi du poète que sur ses œuvres. Les variantes tessitures (masculines ou féminines) choisies pour l'incarner témoignent de cette interrogation. De même, la figure historique de Lincoln est-elle convoquée par l'Anglais Sherwood (1938) dans Abe Lincoln in Illinois pour refléter les hésitations d'un homme qui doit s'arracher à la tentation de l'isolement pour incarner son destin ; combat qui, pour Claude Coulon (pp.177-191), fut tant celui de l'homme d'État américain que celui du dramaturge. Identique est l'angle choisi par John Osborne pour représenter en 1961 Martin Luther, dans la pièce analysée par Nicole Boireau (pp. 85-98) : sur scène s'expriment davantage les souffrances d'une âme abritée par un corps malade que la conscience révolutionnaire du réformateur. La réflexion psychologique est particulièrement développée dans les œuvres consacrées aux figures féminines : Cléopâtre, Mary Stuart, Elizabeth Ière sont le lieu d'une interrogation sur le rapport entre féminité et pouvoir, bien sûr, sur l'impasse à laquelle sont condamnées les souveraines, sommées de choisir entre l'amour et les devoirs politiques, mais aussi sur l'identité sexuelle. Jean-Pierre Simard (pp. 129-144) montre comment Mary Stuart est écartelée entre les deux faces duelles de la sainte martyre ou de la catin criminelle, 
tandis que le personnage d'Elizabeth Ière forgé d'après la biographie psychanalytique de Lytton Strachey (1928), peut se lire comme une projection des tourments de Benjamin Britten, homosexuel et objecteur de conscience. L'Adriana Lecouvreur (1902) de Francesco Cilea et Arturo Collauti, étudié par Walter Zidaric (pp. 145-150) grâce à la mise en abyme de la figure de la diva, prolonge cette quête de l'identité féminine, dans le même temps qu'il introduit à la dernière thématique abordée par les œuvres du corpus: la création artistique. Jean-Philippe Heberlé (pp.99-111) met en évidence combien l'opéra de Peter Maxwell Davies, daté de 1972, Taverner, est une réflexion sur le travail du musicien et sur les doutes de l'artiste. Quant à Giuseppe Verdi, objet des deux articles de Myriam Gracia (pp. 207-217) et Chantal Cazaux (pp. 69-83), il laissa se construire une biographie mythique fixant l'image de l'artiste en homme politique engagé qui servit sa carrière. Ariane Essein (pp.193-206) montre enfin comment l'interview imaginaire de Charles Dickens par Giorgio Manganelli, radiodiffusée sur la Rai en 1974, se plaît à brouiller les pistes biographiques pour questionner l'auteur, son œuvre et son public : Charles Dickens n'est-il finalement qu'une projection mentale du lecteur, une création de l'écrivain par lui-même, un pur objet intellectuel ?

Le dernier article du recueil, l'analyse consacrée par Raymond Michel (pp. 275-302) au Roberto Zucco de Bernard-Marie Koltès, révèle l'unité profonde de ces études et des œuvres qu'elles explorent : la mythification. Un même mouvement tend à faire de tous les personnages réels des héros, ou des antihéros (certes, certains l'étaient déjà : Macbeth, Napoléon, Cléôpatre, Rimbaud, etc.), en un mouvement du singulier à l'universel que symbolise chez Koltès la réécriture du nom de Roberto Succo. Le parcours initiatique qu'il projette sur la figure du tueur se lirait alors comme une version paradigmatique de toutes les Vies évoquées.

Finalement, qu'elles nous obligent à revisiter nos classiques, nous confrontent à des domaines peu connus ou entrent en résonance avec nos souvenirs cinématographiques, ces études stimulent notre regard de spectateur.

INDEX

oeuvrecitee Réécriture des sources biographiques et construction du personnage de théâtre (Nicole Boireau, Jean-Fréséric Chevalier et Pierre Degott, 2010)

\section{AUTEURS}

\section{LAURENCE BECK-CHAUVARD}

Écritures, université Paul Verlaine-Metz

chauvard@alice.itCulture, esthétique 